PROCEEDINGS OF THE

AMERICAN MATHEMATICAL SOCIETY

Volume 133, Number 10, Pages 3013-3020

S 0002-9939(05)07812-3

Article electronically published on March 24, 2005

\title{
SPECTRA OF UPPER TRIANGULAR OPERATOR MATRICES
}

\author{
C. BENHIDA, E. H. ZEROUALI, AND H. ZGUITTI
}

(Communicated by Joseph A. Ball)

\begin{abstract}
Let $X, Y$ be given Banach spaces. For $A \in \mathcal{L}(X), B \in \mathcal{L}(Y)$ and $C \in \mathcal{L}(Y, X)$, let $M_{C}$ be the operator defined on $X \oplus Y$ by $M_{C}=\left[\begin{array}{c}A \\ 0\end{array}{ }_{B}^{C}\right]$. We give sufficient conditions on $C$ to get $\Sigma\left(M_{C}\right)=\Sigma\left(M_{0}\right)$, where $\Sigma$ runs over a large class of spectra. We also discuss the case of some spectra for which the latter equality fails.
\end{abstract}

\section{INTRODUCTION}

In this paper, $X$ and $Y$ are Banach spaces and $\mathcal{L}(X, Y)$ denotes the space of all bounded linear operators from $X$ to $Y$. We let $\mathcal{L}(X)$ denote $\mathcal{L}(X, X)$. For a bounded linear operator $T \in \mathcal{L}(X)$, let $N(T), R(T), \sigma(T), \sigma_{e}(T), \sigma_{S F}(T), \sigma_{w}(T), \sigma_{a p}(T)$, and $\sigma_{s}(T)$ denote the null space, the range, the spectrum, the essential spectrum, the Semi-Fredholm spectrum, the Weyl spectrum, the approximate point spectrum, and the surjectivity spectrum of $T$, respectively.

Let $D(\lambda, r)$ be the open disk centred at $\lambda \in \mathbb{C}$ and with radius $r>0$. The corresponding closed disc will be denoted by $\bar{D}(\lambda, r)$. For an open set $U$ in $\mathbb{C}$, we denote by $\mathcal{O}(U, X)$ and $\mathcal{E}(U, X)$ the Fréchet space of all $X$-valued analytic functions on $U$ and the Fréchet space of all $X$-valued $C^{\infty}$-functions on $U$, respectively.

A bounded linear operator $T \in \mathcal{L}(X)$ is said to have the single-valued extension property ( $S V E P$ for short) at $\lambda \in \mathbb{C}$ if there exists $r>0$ such that for every open subset $U \subset D(\lambda, r)$, the only analytic solution of the equation $(T-\mu) x(\mu)=0$ is the null function. We use $\mathcal{S}(T)$ to denote the set where $T$ fails to have the $S V E P$, and we say that $T$ has the $S V E P$ when $\mathcal{S}(T)$ is the empty set, 11].

An operator $T$ is said to satisfy Bishop's property $(\beta)$ at $\lambda \in \mathbb{C}\left(\operatorname{resp} .(\beta)_{\epsilon}\right)$ if there exists $r>0$ such that for every open subset $U \subset D(\lambda, r)$ and for any sequence $\left(f_{n}\right)_{n}$ in $\mathcal{O}(U, X)$ (resp. in $\left.\mathcal{E}(U, X)\right)$ such that whenever $(T-\mu) f_{n}(\mu) \longrightarrow 0$ in $\mathcal{O}(U, X)$ (resp. in $\mathcal{E}(U, X))$, then $f_{n}(\mu) \longrightarrow 0$ in $\mathcal{O}(U, X)$ (resp. in $\mathcal{E}(U, X)$ ). Let $\sigma_{\beta}(T)$ (resp. $\sigma_{\beta_{\epsilon}}(T)$ ) be the set of all points where $T$ does not have property $(\beta)$ (resp. $\left.(\beta)_{\epsilon}\right)$. Then $T$ is said to satisfy Bishop's property $(\beta)$ (resp. $\left.(\beta)_{\epsilon}\right)$ precisely when $\sigma_{\beta}(T)=\emptyset\left(\right.$ resp. $\left.\sigma_{\beta_{\epsilon}}(T)=\emptyset\right)$.

The operator $T$ is said to have the spectral decomposition property $(\delta)$ at $\lambda$ if there exists an open neighbourhood $U$ of $\lambda$ such that for every finite open cover $\left\{U_{1}, \ldots, U_{n}\right\}$ of $\mathbb{C}$, with $\sigma(T) \backslash U \subseteq U_{1}$, we have

$$
\mathcal{X}_{T}\left(\bar{U}_{1}\right)+\cdots+\mathcal{X}_{T}\left(\bar{U}_{n}\right)=X,
$$

Received by the editors February 26, 2004 and, in revised form, May 18, 2004.

2000 Mathematics Subject Classification. Primary 47A11, 47A10.

Key words and phrases. Local spectral theory, operator matrices, spectra. 
where $\mathcal{X}_{T}(F)$ is the set of elements $x \in X$ such that the equation $(T-\lambda) x(\lambda)=x$ has a global analytic solution on $\mathbb{C} \backslash F$. Following [13, page 32], $\mathcal{X}_{T}(F)$ is called the glocal analytic spectral subspace associated with $F$, since the analytic functions in their definition are globally defined on $\mathbb{C} \backslash F$, but will depend on $x$.

If moreover in Equation (1) the glocal subspaces are closed, then $T$ is said to be decomposable at $\lambda$.

The $\delta$-spectrum $\sigma_{\delta}(T)$ and the decomposability spectrum $\sigma_{d e c}(T)$ are defined in a similar way.

The properties $(\beta)$ and $(\delta)$ are known to be dual to each other in the sense that $\sigma_{\delta}(T)=\sigma_{\beta}\left(T^{*}\right)$. An operator is decomposable at $\lambda$ if it has both $(\beta)$ and $(\delta)$ at $\lambda$. Thus $\sigma_{\text {dec }}(T)=\sigma_{\delta}(T) \cup \sigma_{\beta}(T)$. We refer to [1, 5, 6, 13] for a complete study of local spectral theory and further details.

For $A \in \mathcal{L}(X), B \in \mathcal{L}(Y)$ and $C \in \mathcal{L}(Y, X)$ we denote by $M_{C}$ the operator defined on $X \oplus Y$ by

From the factorisation formula

$$
M_{C}=\left[\begin{array}{cc}
A & C \\
0 & B
\end{array}\right]
$$

$$
\left[\begin{array}{cc}
A & C \\
0 & B
\end{array}\right]=\left[\begin{array}{ll}
I & 0 \\
0 & B
\end{array}\right]\left[\begin{array}{cc}
I & C \\
0 & I
\end{array}\right]\left[\begin{array}{cc}
A & 0 \\
0 & I
\end{array}\right]
$$

it is not hard to see that for every $C$, we have $\sigma\left(M_{C}\right) \subset \sigma(A) \cup \sigma(B)$. The latter inclusion may well be strict as shown for $A=S, B=S^{*}$ and $C=I-S S^{*}$, where $S$ is the usual shift operator on the Hardy space. On the other hand, this inclusion turns out to be an equality in the case where $X$ and $Y$ are finite dimensional.

Numerous mathematicians were interested in the following equality:

$$
\sigma\left(M_{C}\right)=\sigma(A) \cup \sigma(B) . \quad \text { for every } C \in \mathcal{L}(Y, X) .
$$

See for instance [3, 9, 12, 17]. It is in particular known that if $A$ or $B$ is normal or more generally if $A^{*}$ or $B$ has the $S V E P$, then Equation (3) holds.

If for $A$ and $B$ given, Equation (3) is not valid for some $C$, it is natural to study the class of all operators $C$ such that $\sigma\left(M_{C}\right)=\sigma(A) \cup \sigma(B)$. This has motivated M. Barraa and M. Boumazgour in [3] where the main result is:

Theorem 1.1 ([3, Theorem 1]). If $A \in \mathcal{L}(X)$ and $B \in \mathcal{L}(Y)$, then

$$
\begin{gathered}
\sigma\left(M_{C}\right)=\sigma(A) \cup \sigma(B) \\
\text { for every } C \in \operatorname{cl}\left[R\left(\delta_{A, B}\right)+N\left(\delta_{A, B}\right)+\bigcup_{\lambda \in \mathbb{C}} N\left(L_{A-\lambda}\right)+\bigcup_{\lambda \in \mathbb{C}} N\left(R_{B-\lambda}\right)\right] .
\end{gathered}
$$

Here $L_{A}$ (resp. $R_{B}$ ) is the left (resp. right) multiplication operator given by $L_{A}(X)=A X\left(\right.$ resp. $\left.R_{B}(X)=X B\right)$, and $\delta_{A, B}=L_{A}-R_{B}$ is the usual generalized derivation associated with $A$ and $B$. The symbol $c l[$.$] denotes as usual the closure.$

In this paper, we extend Theorem 1.1 in two directions. We substitute the spectrum by spectra with some universal properties. This provides analogues of Theorem 1.1 for a large classe of spectra. We also extend the class of operators $C$ for which Equation (3) is valid. Our tools are some basics from local spectral theory and the $R S-S R$ technique used in [2, 4, 17].

\section{Spectral properties of $M_{C}$}

For $T$ a bounded operator, let $N^{\infty}(T)=\bigcup_{n \geq 1} N\left(T^{n}\right)$ denote the generalized kernel of $T$. 
We start with the next result.

Theorem 2.1. Assume in (a)-(d) below that $S, R, T$ and $N$ are bounded linear operators on a Banach space. Let $\Sigma$ be a "spectrum" with the following properties:

(a) $\Sigma(R S)=\Sigma(S R)$;

(b) for all $T$ and all $N$ such that $T N=N T$ and $N^{2}=0, \Sigma(T+N)=\Sigma(T)$;

(c) for all $T$ and any $\alpha \in \mathbb{C}, \Sigma(T+\alpha)=\Sigma(T)+\alpha$;

(d) the map $T \rightarrow \Sigma(T)$ is upper semicontinuous.

Then, whenever $A \in \mathcal{L}(X), B \in \mathcal{L}(Y)$ and $C$ is in the closure of $\left[R\left(\delta_{A, B}\right)+\right.$ $\left.N\left(\delta_{A, B}\right)+\bigcup_{\lambda \in \mathbb{C}} N^{\infty}\left(L_{A-\lambda}\right)+\bigcup_{\lambda \in \mathbb{C}} N^{\infty}\left(R_{B-\lambda}\right)\right]$, we have

$$
\Sigma\left(M_{C}\right)=\Sigma\left(M_{0}\right) \text {. }
$$

Proof. We split the proof into 3 steps.

Step 1. If $C=C_{2}+C_{1} \in N^{\infty}\left(L_{A-\lambda}\right)+N^{\infty}\left(R_{B-\mu}\right)$, we set

$$
R=\left[\begin{array}{cc}
I & 0 \\
0 & B-\lambda
\end{array}\right] \text { and } S=\left[\begin{array}{cc}
I & C \\
0 & I
\end{array}\right]\left[\begin{array}{cc}
A-\lambda & 0 \\
0 & I
\end{array}\right] \text {. }
$$

Then $R S=M_{C}-\lambda$ and $S R=M_{C(B-\lambda)}-\lambda$. It follows from assumption (a), that

$$
\Sigma\left(M_{C}-\lambda\right)=\Sigma\left(M_{C(B-\lambda)}-\lambda\right)
$$

and (c) implies that

$$
\Sigma\left(M_{C}\right)=\Sigma\left(M_{C(B-\lambda)}\right)
$$

Therefore by induction

$$
\Sigma\left(M_{C}\right)=\Sigma\left(M_{C(B-\lambda)^{n}}\right), \quad \text { for all } n \geq 0 .
$$

Now if we take

$$
R=\left[\begin{array}{cc}
I & 0 \\
0 & B-\mu
\end{array}\right]\left[\begin{array}{cc}
I & C \\
0 & I
\end{array}\right] \text { and } S=\left[\begin{array}{cc}
A-\mu & 0 \\
0 & I
\end{array}\right]
$$

then in a similar way, we get

$$
\Sigma\left(M_{C}\right)=\Sigma\left(M_{(A-\mu)^{m} C}\right), \text { for all } m \geq 1,
$$

and so

$$
\Sigma\left(M_{C}\right)=\Sigma\left(M_{(A-\mu)^{m} C(B-\lambda)^{n}}\right), \text { for all } n, m \geq 1 .
$$

Hence, if $C_{2}+C_{1} \in \bigcup_{\lambda \in \mathbb{C}} N^{\infty}\left(R_{B-\lambda}\right)+\bigcup_{\lambda \in \mathbb{C}} N^{\infty}\left(L_{A-\lambda}\right)$, from (7), we infer that

$$
\Sigma\left(M_{C}\right)=\Sigma\left(M_{0}\right) .
$$

Step 2. Suppose now that $C_{3} \in N\left(\delta_{A, B}\right)$ and write $M_{C_{3}+C_{2}+C_{1}}=M_{C_{2}+C_{1}}+N$, with $N=\left[\begin{array}{cc}0 & C_{3} \\ 0 & 0\end{array}\right]$. Since clearly $N^{2}=0$ and $N M_{C_{2}+C_{1}}=M_{C_{2}+C_{1}} N$, it follows from (b) that $\Sigma\left(M_{C_{3}+C_{2}+C_{1}}\right)=\Sigma\left(M_{C_{2}+C_{1}}\right)$.

Step 3. For $C_{4} \in R\left(\delta_{A, B}\right)$, it is not hard to see that $M_{C_{4}+C_{3}+C_{2}+C_{1}}$ and $M_{C_{3}+C_{2}+C_{1}}$ are similar. Thus, from (a) it follows that

$$
\Sigma\left(M_{C_{4}+C_{3}+C_{2}+C_{1}}\right)=\Sigma\left(M_{C_{3}+C_{2}+C_{1}}\right) .
$$


Finally, if $C_{4}+C_{3}+C_{2}+C_{1} \in\left[R\left(\delta_{A, B}\right)+N\left(\delta_{A, B}\right)+\bigcup_{\lambda, \mu \in \mathbb{C}}\left(N^{\infty}\left(L_{A-\lambda}\right)+\right.\right.$ $\left.\left.N^{\infty}\left(R_{B-\mu}\right)\right)\right]$, we obtain

$$
\Sigma\left(M_{C_{4}+C_{3}+C_{2}+C_{1}}\right)=\Sigma\left(M_{0}\right) .
$$

We complete the proof by appealing to the upper semicontinuity of the spectral mapping $T \rightarrow \Sigma(T)$.

Remarks. (1) Property (a) has been studied in several papers and turns out to be true for $\mathcal{S}, \sigma_{\beta}, \sigma_{\beta_{\epsilon}}, \sigma_{\delta}$ and $\sigma_{d e c}$ in general. If moreover $S$ or $R$ are supposed to be normal or injective with dense range, then (a) holds also for the spectrum, left and right spectrum and for the corresponding essential spectra. We refer to [2, 4, 16] for more details.

(2) Assumptions (c) and (d) in Theorem 2.1 are satisfied for a large class of spectra. They are well known and are usually obtained by straightforward calculations.

(3) To check (b) for the spectrum, left or right spectrum and the corresponding essential spectra, it suffices to compute the inverse of $(T+N-\lambda)$ in terms of the inverse of $(T-\lambda)$. This property for spectra provided by local spectral theory is less well known. We assemble in the following lemma some spectra for which (b) is valid.

Lemma 2.1. Let $T$ be a bounded operator and $N$ an operator commuting with $T$ such that $N^{2}=0$. Then

$$
\Sigma(T+N)=\Sigma(T)
$$

for $\Sigma \in\left\{\sigma, \sigma_{e}, \sigma_{w}, \sigma_{S F}, \sigma_{a p}, \sigma_{s}, \mathcal{S}, \sigma_{\beta}, \sigma_{\beta_{\epsilon}}, \sigma_{\delta}, \sigma_{d e c}\right\}$.

Proof. Because of symmetry, only one inclusion is needed. We treat the case $\sigma_{\beta}(T)$. The claim for the other spectra arising from local spectral theory is obtained either in a similar way or as a corollary. Suppose that $\lambda \notin \sigma_{\beta}(T)$ and let $\mathcal{V}$ be a neighbourhood of $\lambda$. If $f_{n}$ is any sequence of analytic $X$-valued functions such that $(T+N-\mu) f_{n}(\mu)$ converges to zero on compact sets, then $(T-\mu) N f_{n}(\mu)=$ $N(T+N-\mu) f_{n}(\mu)$ converges to zero on compact sets, and so is $N f_{n}$. This clearly leads to $(T-\mu) f_{n}(\mu)$ converging to zero on compact sets. Again, from $\lambda \notin \sigma_{\beta}(T)$, we conclude that $f_{n}$ converges to zero on compact sets. Finally $\lambda \notin \sigma_{\beta}(T+N)$.

(4) The mapping $T \rightarrow \mathcal{S}(T)$ is not upper semicontinuous. However, since (a)(c) are satisfied in this setting, we deduce that $\mathcal{S}\left(M_{C}\right)=\mathcal{S}\left(M_{0}\right)$ for every $C \in$ $\left[R\left(\delta_{A, B}\right)+N\left(\delta_{A, B}\right)+\bigcup_{\lambda \in \mathbb{C}} N^{\infty}\left(L_{A-\lambda}\right)+\bigcup_{\lambda \in \mathbb{C}} N^{\infty}\left(R_{B-\lambda}\right)\right]$.

As a consequence of Theorem 2.1, we get

Corollary 2.1. Let $A, B$ and $C$ be as in Theorem 2.1. Then $M_{C}$ has Bishop's property $(\beta)$ (resp. property $(\delta)$, resp. is decomposable) if and only if both $A$ and $B$ satisfy Bishop's property $(\beta)$ (resp. property $(\delta)$, resp. are decomposable).

For $\Sigma \subset \mathbb{C}$, we set $\Sigma_{*}=\Sigma \backslash\{0\}$.

Theorem 2.2. Let $S, R, T, N$ and $\Sigma$ satisfy the assumptions (b)-(d) of Theorem 2.1 and suppose that

$\left(\mathrm{a}_{*}\right) \Sigma_{*}(R S)=\Sigma_{*}(S R)$.

Then, whenever $A \in \mathcal{L}(X), B \in \mathcal{L}(Y)$ and $C \in \operatorname{cl}\left[R\left(\delta_{A, B}\right)+N\left(\delta_{A, B}\right)+\right.$ $\left.\bigcup_{\lambda \in \mathbb{C}} N^{\infty}\left(L_{A-\lambda}\right)+\bigcup_{\lambda \in \mathbb{C}} N^{\infty}\left(R_{B-\lambda}\right)\right]$, we have

$$
\Sigma_{*}\left(M_{C}\right)=\Sigma_{*}\left(M_{0}\right) .
$$




\section{If moreover,}

(e) for all $T$ and all $N$ such that $T N=0$ (or $N T=0$ ) and $N^{2}=0$ we have $\Sigma(T+N)=\Sigma(T)$

then $\Sigma\left(M_{C}\right)=\Sigma\left(M_{0}\right)$.

Proof. We proceed as in the previous theorem. The possible changes are in the first step, since condition $\left(\mathrm{a}_{*}\right)$ only gives $\Sigma_{*}\left(M_{C_{2}+C_{1}}\right)=\Sigma_{*}\left(M_{0}\right)$. We set $N_{C}=$ $\left(\begin{array}{ll}0 & C \\ 0 & 0\end{array}\right)=0$. Then obviously $N_{C}^{2}=0,\left(M_{0}-\lambda\right) N_{C}=0$ if $C \in N\left(L_{A}-\lambda\right)$ and $N_{C}\left(M_{0}-\lambda\right)=0$ if $C \in N\left(R_{B}-\lambda\right)$. We derive from properties (c) and (e) that $\Sigma\left(M_{C}\right)=\Sigma\left(M_{0}\right)$ for every $C \in \bigcup_{\lambda \in \mathbb{C}} N^{\infty}\left(L_{A-\lambda}\right)+\bigcup_{\lambda \in \mathbb{C}} N^{\infty}\left(R_{B-\lambda}\right)$.

We then end the proof as in Theorem 2.1.

Remark. In Theorem 2.2 it is not generally possible to get $\Sigma\left(M_{C}\right)=\Sigma\left(M_{0}\right)$. The purpose of the following example is to show this fact.

Example 1. Let $A=S$ be the shift operator on the Hardy space $H^{2}, B=I-e_{0} \otimes e_{0}$ and $C=e_{0} \otimes e_{0}$ where $e_{0}$ is the first element of the canonical orthonormal basis of $H^{2}$. Then clearly $C B=0$ and $\sigma_{a p}\left(M_{0}\right)=\{z \in \mathbb{C}:|z|=1\} \cup\{0\}$. Let us prove that $0 \notin \sigma_{a p}\left(M_{C}\right)$. Suppose that for some $\left(x_{n}, y_{n}\right)$, we have $\lim _{n \rightarrow \infty} M_{C}\left(x_{n}, y_{n}\right)=0$. Then

$$
\begin{cases}\lim _{n \rightarrow \infty}\left(S x_{n}+e_{0} \otimes e_{0}\left(y_{n}\right)\right) & =0, \\ \lim _{n \rightarrow \infty}\left(I-e_{0} \otimes e_{0}\right) y_{n} & =0 .\end{cases}
$$

Now since $R(S) \perp \mathbb{C} e_{0}$, we deduce that $\lim _{n \rightarrow \infty}\left(S x_{n}\right)=\lim _{n \rightarrow \infty}\left(e_{0} \otimes e_{0}\right) y_{n}=0$. Then $\lim _{n \rightarrow \infty} y_{n}=\lim _{n \rightarrow \infty}\left(e_{0} \otimes e_{0}\right) y_{n}+\lim _{n \rightarrow \infty}\left(I-e_{0} \otimes e_{0}\right) y_{n}=0$ and $\lim _{n \rightarrow \infty} x_{n}=\lim _{n \rightarrow \infty} S^{*} S x_{n}=0$.

We recall that for $x \in X$, the resolvent set $\rho_{T}(x)$ of $T$ at $x$ is defined as follows: A complex number $\lambda$ is in $\rho_{T}(x)$ if there exists a neighborhood $O$ of $\lambda$ and $f: O \rightarrow X$, an analytic function, such that $(T-\mu) f(\mu)=x$ for every $\mu \in O$. The local spectrum of $T$ at $x$ is then given by $\sigma_{T}(x)=\mathbb{C} \backslash \rho_{T}(x)$. One may expect to get similar results for local spectra. To be precise, if $C$ is as in Theorem 2.1, does the following equality hold:

$$
\sigma_{M_{C}}(x, y)=\sigma_{A}(x) \cup \sigma_{B}(y) ?
$$

This is not always true as shown by the example below.

Example 2. Let $A=S$ be the shift operator on the Hardy space $H^{2}, B=S^{*}$ and $C=A-B \in R\left(\delta_{A, B}\right)$. Take $(x, y)=\left(0, e_{0}\right)$ where $e_{0}$ is the first element of the basis. Since $\sigma_{A}(0)=\sigma_{B}\left(e_{0}\right)=\emptyset$, we get $\sigma_{A}(x) \cup \sigma_{B}(y)=\emptyset$. On the other hand, if $\lambda_{0} \notin \sigma_{M_{C}}(x, y)$, then there exist $x(\lambda)$ and $y(\lambda)$ analytic in a neighbourhood of $\lambda_{0}$ such that

$$
\left\{\begin{array}{lll}
(A-\lambda) x(\lambda)+C y(\lambda) & =0 \\
(B-\lambda) y(\lambda) & =e_{0} .
\end{array}\right.
$$

In particular $(A-\lambda)(x(\lambda)+y(\lambda))=e_{0}$; hence $\sigma_{A}\left(e_{0}\right) \neq \sigma(A)$. Now because $\sigma_{A}(x)=\sigma(A)=\bar{D}(0,1)$ for every $x \neq 0$, we obtain a contradiction.

In contrast with the example above, we have

Theorem 2.3. Let $A$ and $B$ be given. If $C \in N\left(R_{B}^{n}\right)$ for some $n \geq 1$, then for every $x \oplus y \in X \oplus Y$ we have

$$
\sigma_{M_{C}}\left(x \oplus B^{n} y\right) \subset \sigma_{A}(x) \cup \sigma_{B}(y) .
$$


If in addition $B$ is injective, we get

$$
\sigma_{M_{C}}\left(x \oplus B^{n} y\right)=\sigma_{A}(x) \cup \sigma_{B}(y) .
$$

Proof. From the decomposition $R=\left[\begin{array}{cc}I & 0 \\ 0 & B\end{array}\right]$ and $S=\left[\begin{array}{cc}I & C \\ 0 & I\end{array}\right]\left[\begin{array}{cc}A & 0 \\ 0 & I\end{array}\right]$ and using [4, Proposition 3.1 (ii)] we obtain

$$
\begin{aligned}
\sigma_{M_{C}}\left(x \oplus B^{n} y\right) & \subset \sigma_{M_{C B}}\left(x \oplus B^{n-1} y\right) \\
& \subset \sigma_{M_{C B^{2}}}\left(x \oplus B^{n-2} y\right) \\
& \cdot \\
& \cdot \\
& \subset \sigma_{M_{C B} n}(x \oplus y)=\sigma_{A}(x) \cup \sigma_{B}(y) .
\end{aligned}
$$

Now if $B$ is injective the equality follows from [4. Proposition 3.1].

\section{Applications and CONClUding REMARKS}

3.1. Weyl's theorem. H. Weyl 18 has shown that every hermitian operator $T \in$ $\mathcal{L}(X)$ satisfies

$$
\sigma(T) \backslash \sigma_{\omega}(T)=\pi_{00}(T),
$$

where $\pi_{00}(T)$ consists of the isolated points of $\sigma(T)$ which are eigenvalues of finite multiplicity.

We say that Weyl's theorem holds for an operator $T \in \mathcal{L}(X)$ if Equation (9) holds. Weyl's theorem has been extended to several classes of operators including normals and hyponormals.

Let $A$ and $B$ be given. In general, the fact that Weyl's theorem holds for $M_{0}$ $(=A \oplus B)$ does not imply that Weyl's theorem holds for $M_{C}$; see for instance [14]. It also may happen that $M_{C}$ satisfies Weyl's theorem while $M_{0}$ does not satisfy it. We consider the following example to show the last claim. If $A=B^{*}$ is the shift operator and $C=I-S S^{*}$, then $M_{C}$ is unitary without eigenvalues and hence satisfies Weyl's theorem. Now $\sigma_{w}\left(M_{0}\right)=\{z:|z|=1\}$ and $\sigma\left(M_{0}\right) \backslash \pi_{00}(T)=\bar{D}$. Thus $M_{0}$ does not satisfy Weyl's theorem.

By [4, Proposition 4.4] and Theorem [2.2 we get

Proposition 3.1. Let $A, B$ be given, and suppose that $0 \in \Pi_{00}\left(M_{C}\right) \cap \Pi_{00}\left(M_{0}\right)$ or $0 \notin \Pi_{00}\left(M_{C}\right) \cup \Pi_{00}\left(M_{0}\right)$, for some $C \in \operatorname{cl}\left[R\left(\delta_{A, B}\right)+N\left(\delta_{A, B}\right)+\bigcup_{\lambda \in \mathbb{C}} N^{\infty}\left(L_{A-\lambda}\right)+\right.$ $\left.\bigcup_{\lambda \in \mathbb{C}} N^{\infty}\left(R_{B-\lambda}\right)\right]$. Then Weyl's theorem holds for $M_{0}$ if and only if Weyl's theorem holds for $M_{C}$.

Proof. For the obvious symmetry reason we shall show only one way. In [4 Proposition 4.4], it is shown that if $0 \in \pi_{00}(R S) \cap \pi_{00}(S R)$ or $0 \notin \pi_{00}(R S) \cup \pi_{00}(S R)$, then Weyl's theorem holds for $R S$ if and only if Weyl's theorem holds for $S R$. Now suppose Weyl's theorem holds for $A \oplus B$. We argue as in Step 1 of the proof of Theorem 2.2 to see that Weyl's theorem holds for $M_{C_{2}+C_{1}}$, when $C_{2}+C_{1} \in$ $\bigcup_{\lambda \in \mathbb{C}} N^{\infty}\left(L_{A-\lambda}\right)+\bigcup_{\lambda \in \mathbb{C}} N^{\infty}\left(R_{B-\lambda}\right)$. Now, since $M_{C_{3}+C_{2}+C_{1}}=M_{C_{2}+C_{1}}+N$, with $N=\left[\begin{array}{cc}0 & C_{3} \\ 0 & 0\end{array}\right]$ nilpotent and $N M_{C_{2}+C_{1}}=M_{C_{2}+C_{1}} N$, for $C_{3} \in N\left(\delta_{A, B}\right)$, it follows from [15, Theorem 3] that Weyl's theorem holds for $M_{C_{3}+C_{2}+C_{1}}$. Finally $M_{C_{4}+C_{3}+C_{2}+C_{1}}$ and $M_{C_{3}+C_{2}+C_{1}}$ are similar, for $C_{4} \in R\left(\delta_{A, B}\right)$ yields Weyl's theorem for $M_{C}$. 
3.2. Concluding remarks. 1) It would be of some interest to characterise those operators such that

$$
c l\left[R\left(\delta_{A, B}\right)+N\left(\delta_{A, B}\right)+\bigcup_{\lambda \in \mathbb{C}}\left(N^{\infty}\left(L_{A-\lambda}\right)+\bigcup_{\lambda \in \mathbb{C}}\left(N^{\infty}\left(R_{B-\lambda}\right)\right]=\mathcal{L}(H) .\right.\right.
$$

A sufficient condition is given by the Rosenblum-Davis-Rosenthal Corollary, 8 , Corollary 3.2.2], that is, $\sigma_{s}(A) \cap \sigma_{a p}(B)=\emptyset$. Notice here that since $\mathcal{S}\left(A^{*}\right) \cap \mathcal{S}(B) \subset$ $\sigma_{s}(A) \cap \sigma_{a p}(B)$, Equation (10) is immediate from [9].

We also mention here a result of L. Fialkow, [7, Theorem 1.1]: $R\left(\delta_{A, B}\right)$ is dense if and only if $\sigma_{r e}(A) \cap \sigma_{l e}(B)=\emptyset$ and $N\left(\delta_{B, A}\right)$ contains a nonzero trace class operator. Thus, under the last assumption, Equation (10) holds.

2) We finally note that for $\Sigma=\sigma_{w}$, we may have $\Sigma\left(M_{0}\right) \neq \Sigma(A) \cup \Sigma(B)$, as for the example above. However for $\Sigma \in\left\{\sigma, \sigma_{e}, \sigma_{a p}, \sigma_{s}, \sigma_{\beta}, \sigma_{\beta_{\epsilon}}, \sigma_{\delta}, \sigma_{d e c}\right\}$ it is not hard to see that $\Sigma\left(M_{0}\right)=\Sigma(A) \cup \Sigma(B)$. In particular Theorem 1.1 is recaptured for all these spectra.

\section{ACKNOWLEDGEMENT}

The authors are indebted to the referee for several helpful remarks and suggestions on a former version of this paper.

A part of this paper was written while the second author was visiting the University of Lille 1.

\section{REFERENCES}

[1] E. Albrecht and J. Eschmeier, Analytic functional models and local spectral theory. Proc. London Math. Soc. (3), 75(1997), 323-348. MR1455859 (98f:47043)

[2] B. Barnes, Common operator properties of the linear operators $R S$ and $S R$. Proc. Amer. Math. Soc. 126(1998), 1055-1061. MR1443814 (98f:47003)

[3] M. Barraa and M. Boumazgour, A note on the spectrum of an upper triangular operator matrix. Proc. Amer. Math. Soc. 131(2003), 3083-3088. MR1993217 (2004d:47009)

[4] C. Benhida and E. H. Zerouali, Local spectral theory of linear operators $R S$ and $S R$. Preprint.

[5] E. Bishop, A duality theorem for an arbitrary operator. Pacific J. Math. 9(1959), 379-397. MR0117562(22:8339)

[6] I. Colojoară and C. Foiaş, Theory of generalized spectral operators. Gordon and Breach, New York 1968. MR0394282 (52:15085)

[7] L. A. Fialkow, A note on the range of the operator $X \rightarrow A X-X B$. Illinois J. Math. 25 (1981), 112-124. MR0602902 (84b:47021)

[8] D. A. Herrero, Approximation of Hilbert space operators. Vol. 1. Pitman Research Notes in Mathematics Series, 224. New York, 1989. MR1088255 (91k:47002)

[9] H. Elbjaoui and E. H. Zerouali, Local spectral theory for $2 \times 2$ operator matrices. Int. J. Math and Mathematical Sciences, 42(2003), 2667-2672. MR.2005905 (2004f:47006)

[10] J. Eschmeier and M. Putinar, Bishop's condition $(\beta)$ and rich extensions of linear operators. Indiana Univ. Math. J. 37(1988), 325-348. MR0963505 (89k:47051)

[11] J. K. Finch, The single valued extension property on a Banach space. Pacific J. Math. 58 (1975), 61-69. MR0374985 (51:11181)

[12] M. Houimdi and H. Zguitti, Propriétés spectrales locales d'une matrice carrée des opérateurs. Acta Math. Vietnam. 25(2000), 137-144. MR.1770883 (2001d:47011)

[13] K. B. Laursen and M. M. Neumann, An introduction to local spectral theory. Clarendon, Oxford, 2000. MR1747914 (2001k:47002)

[14] W. Y. Lee, Weyl spectra for operator matrices. Proc. Amer. Math. Soc. 129(2000), 131-138. MR 1784020 (2001f:47003)

[15] K. K. Oberai, On the Weyl's spectrum (II), Illinois. J. Math. 21(1977), 84-90. MR0428073 $(55: 1102)$ 
[16] C. Lin, Y. Zikun and R. Yingbin, Common properties of operators $R S$ and $S R$ and p-hyponormal operators. Int. Eq. Op. Th. 43(2002), 313-325. MR.1902952 (2003b:47041)

[17] E. H. Zerouali and H. Zguitti, Perturbation of spectra of operator matrices and local spectral theory. Preprint.

[18] H. Weyl, Über beschränkte quadratische formen, deren Differenz vollsteig ist. Rend. Circ. Mat. Palermo 27(1909) 373-392.

UFR de Mathématiques - CNRS-UMR 8524, Université de Lille 1, BÂt M2, 59655 Villeuneuve Cedex, France

E-mail address: benhida@math.univ-lille1.fr

Département de Mathématiques et Informatique, Faculté des Sciences de Rabat, BP 1014 Agdal, Rabat, Maroc

E-mail address: zerouali@fsr.ac.ma

Département de Mathématiques et Informatique, Faculté des Sciences de Rabat, BP 1014 AgDal, Rabat, Maroc

E-mail address: zguitti@hotmail.com 